\title{
Les nouvelles frontières de la laïcité : la conquête de l'Ouest?
}

\section{Stéphanie Hennette Vauchez}

\section{(2) OpenEdition}

12 Journals

Édition électronique

URL : https://journals.openedition.org/rdr/666

DOI : $10.4000 /$ rdr.666

ISSN : 2534-7462

Éditeur

Presses universitaires de Strasbourg

\section{Édition imprimée}

Date de publication : 31 octobre 2017

Pagination : 19-32

ISBN : 978-2-86820-974-0

ISSN : 2493-8637

Référence électronique

Stéphanie Hennette Vauchez, "Les nouvelles frontières de la laïcité : la conquête de l'Ouest ? ", Revue du droit des religions [En ligne], 4 | 2017, mis en ligne le 15 janvier 2020, consulté le 23 mai 2022. URL http://journals.openedition.org/rdr/666 ; DOI : https://doi.org/10.4000/rdr.666

\section{(c) (7) (9)}

La revue du droit des religions est mise à disposition selon les termes de la Creative Commons Attribution - Pas d'Utilisation Commerciale 4.0 International - CC BY-NC 4.0. 


\section{LES NOUVELLES FRONTIÈRES DE LA LAÏCITÉ : LA CONQUÊTE DE L'OUEST ?}

\section{Stéphanie HENNETTE VAUCHEZ}

Université Paris Ouest Nanterre, Centre de théorie et analyse du droit (CTAD)

\section{RÉSUMÉ}

Le présent article entend opérer un retour critique sur un peu plus d'une décennie d'évolutions de la portée juridique du principe de laïcité, lu ici comme une frontière à la fois spatiale (ce sont toujours plus d'espaces : l'école, l'espace public, l'entreprise...) et matérielle (l'axiologisation de la laïcité comme ligne de démarcation entre les personnes et groupes de personnes qu'elle sépare). Ce faisant, il suggère que la laïcité est devenue porteuse de valeurs affirmées, la laïité rompt avec la neutralité et se fait programme normatif.

\section{ABSTRACT}

This paper provides a critical approach of the evolutions of the scope of the legal principle of laïcité that have occurred for over a decade. Laïcité is here analysed as spatial frontier as it applies to more and more spaces : school, public space, private companies... It is also considered as a material frontier in that laïcité has become a value-based dividing line between various categories of individuals or groups. This paper argues that, as a result, laïcité now conveys strong values : it breaks with neutrality and becomes a normative programme. 
$\mathrm{N}$ ombre de travaux, dans des champs disciplinaires divers, établissent depuis plusieurs années le passage de la laïcité comme principe d'organisation des pouvoirs publics ${ }^{1}$ à une laïcité axiologique, devenue valeur à respecter ${ }^{2}$. Cette transformation prend appui sur des évolutions majeures de la signification juridique (i.e. des contours et du contenu) du principe de laïcité. En particulier, on observe que les personnes privées sont en effet tendanciellement attraites dans le champ d'application de l'obligation de neutralité religieuse découlant de la laïcité ${ }^{3}$ - ce qui tranche fortement avec un état antérieur du droit, résumé en 1949 par Jean Rivero au moyen d'une formule simple définissant la laïcité comme "la neutralité des personnes publiques ${ }^{4}$ », et confirmé en 2004 par le Conseil d'État dans une vaste étude portant sur Un siècle de laïcité $e^{5}$ : l'obligation de neutralité religieuse générée par le principe juridique de laïcité ne concerne que les personnes publiques ; pour les personnes privées, au contraire, ce principe emporte (comprend, englobe) la liberté de conscience et, corrélativement, la liberté de culte et donc, d'exprimer sa foi.

Or, ce processus de redéfinition de la laïcité peut avantageusement être analysé du point de vue spatial. En effet, les personnes privées ont été progressivement soumises à une obligation de neutralité religieuse dans un nombre croissant de cas, non pas en tant que telles, mais en fonction des lieux où elles se trouvent : ainsi, à l'école, autour de l'école, au travail parfois, dans la rue dans une certaine mesure..., on a vu apparaître des règles juridiques prescrivant la neutralité religieuse des personnes. Il y a donc de nouvelles frontières de la laïcité qui se dessinent à mesure que l'obligation de neutralité religieuse se requiert aujourd'hui dans un toujours plus grand nombre d'espaces. On peut même tenter un parallèle avec la conquête du Far West qui marquait, au $\mathrm{XIX}^{\mathrm{e}}$ et jusqu'au début du $\mathrm{XX}^{\mathrm{e}}$ siècle, la construction des États-Unis d'Amérique tels qu'on les connaît aujourd'hui, pour considérer qu'il y a une conquête de la laïcité ainsi appréhendée comme frontière - frontière sans cesse renouvelée et repoussée. Certes, il ne s'agirait pas pour autant d'une conquête de l'Ouest ; mais les nouvelles frontières de la laïcité participent bien de quelque chose

1. Par ex., Prélot P.-H., « L'interdiction de la dissimulation du visage à la lumière du principe de laïcité », Revue du droit des religions, n² 2, nov. 2016, p. 11.

2. V. en dernier lieu PORTIER Ph., L'État et les religions en France. Une sociologie historique de la laïcité, Presses universitaires de Rennes, 2016.

3. Pour une mise en panorama qui insiste sur cette distinction entre une laïcité qui génère des obligations vis-à-vis des personnes publiques et une laïcité nouvelle qui oblige les personnes privées, on se permet de renvoyer à Hennette Vauchez S., Valentin V., L'affaire Baby Loup ou la nouvelle laïcité, Paris, Lextenso, 2014.

4. Rivero J., « La laïcité », D. 1949, chron. 33.

5. France. Conseil d'État, Un siècle de laïcité, Paris, La Documentation française, 2004. 
comme une conquête par l'Ouest, au sens d'une conquête par laquelle l'Ouest s'affirme et se réaffirme en s'opposant à un autre qu'il construit par cette opposition même, tout en cherchant à le domestiquer, le cantonner voire, parfois, l'exclure. En effet, les frontières ne se bornent pas à distinguer les espaces qu'elles séparent ; plus avant, elles les définissent comme fondamentalement étrangers et disent, ce faisant, pourquoi ils doivent être séparés. En ce sens, il est intéressant de se pencher non seulement sur les aspects physiques (la conquête, les nouveaux espaces), mais aussi politiques ou matériels de ces nouvelles frontières de la laïcité (les identités ainsi produites).

\section{LES FRONTIÈRES DE LA LAÏCITÉ : CARTOGRAPHIE D'UNE CONQUÊTE}

Au cours, grosso modo, de la dernière décennie, on a vu la laïcité devenir le vecteur d'obligations de neutralité religieuse pesant sur les personnes privées. C'est le cas dans un nombre croissant d'espaces dont on peut dans un premier temps établir la cartographie : cette laïcité-neutralité est aujourd'hui exigée non seulement des personnes publiques mais aussi des personnes privées : à l'école, autour de l'école, parfois dans l'emploi, voire dans la rue.

\section{À L'ÉCOLE ET AUTOUR DE L'ÉCOLE}

Les signes religieux sont désormais interdits à l'école, la chose est entendue. Depuis l'entrée en vigueur de la loi du 15 mars 2004, « le voile islamique, quel que soit le nom qu'on lui donne, la kippa ou une croix de dimension manifestement excessive ${ }^{6} »$ sont proscrits dans les écoles, collèges et lycées publics. On ne reviendra pas ici sur le texte lui-même, les raisons qui ont été formulées à son soutien ou la manière dont il est appliqué ${ }^{7}$. Prolongeant la réflexion sur la notion de frontière, on souhaitera plutôt attirer l'attention sur la manière dont elle a, depuis, été interprétée : alors même que la formulation

6. Circulaire du 18 mai 2004 relative à la mise en œeuvre de la loi n 2004-228 du 15 mars 2004 encadrant, en application du principe de laïcité, le port de signes ou de tenues manifestant une appartenance religieuse dans les écoles, collèges et lycées publics, NOR : MENG0401138C.

7. Pour deux types de bilans bien différents, on pourra lire : Moreau C., « Bilan de l'application de la loi du 15 mars 2004 encadrant, en application du principe de laïcité, le port de signes ou tenues manifestant une appartenance religieuse ", in France. OBSERVATOIRE DE LA LAİcité, Rapport annuel 2013-2014, p. 62 ; Bui-XuAN O., « Regard genré sur les dispositions juridiques relatives à la neutralité religieuse ", in REGINE, HenNETte VAuChez S., Roman D., Möschel M. (dir.), Ce que le genre fait au droit, Paris, Dalloz, 2013, p. 25-45. 
de la loi pouvait laisser présager qu'elle s'appliquait aux personnes que sont les élèves des écoles, collèges et lycées, c'est en fait une construction de l'école comme lieu, et plus encore comme lieu parfaitement homogène, ou pur, du point de vue de l'asepsie religieuse qui le caractérise, qui a émergé de l'interprétation administrative et juridictionnelle de la laïcité promue par la loi de 2004. En d'autres termes, la laïcité a ici dessiné une frontière, une séparation, autour de l'école. On peut donner ici deux illustrations principales.

L'entrée en vigueur de la loi de 2004 a suscité des lectures extensives fondées sur l'idée que c'était l'école comme lieu, et non les élèves en tant qu'usagers du service public, qui étaient assujettis à l'obligation de neutralité religieuse désormais prescrite par la loi. Dans les années qui ont suivi le vote de la loi, certaines écoles entreprenaient de modifier leur règlement intérieur pour assujettir à cette obligation de neutralité les parents accompagnateurs de sorties scolaires, ou encore les usagers de l'école autres que les élèves, et notamment, le cas échéant, les stagiaires de modules de formation professionnelle assurés par des GRETA ${ }^{8}$. Dans les deux cas, ces lectures extensives de l'obligation de neutralité religieuse découlant de la loi de 2004 ont été validées par l'administration scolaire et le gouvernement.

Bien qu'après quelques décisions de justice contrastées ${ }^{9}$, le Conseil d'État a réaffirmé qu'il n'existait aucun fondement juridique permettant d'assujettir des usagers du service public (ici, les parents accompagnateurs) à une obligation de neutralité religieuse et que seuls, le cas échéant, des troubles avérés à l'ordre public seraient de nature à fonder une telle restriction à leur liberté religieuse ${ }^{10}$, la circulaire Chatel de mars $2012^{11}$ prescrivant la neutralité religieuse des accompagnateurs de sorties ${ }^{12}$ demeure en vigueur. En ce sens, le débat illustre le fait que la frontière, conquérante, est aussi disputée.

8. Les GRETA sont « structures de l'Éducation nationale qui organisent des formations pour adultes dans la plupart des métiers ", " groupements d'établissements publics d'enseignement qui mutualisent leurs compétences et leurs moyens pour proposer des formations continues pour adultes $»:$ http://www.education.gouv.fr/cid50753/la-formation-continuedes-adultes-a-l-education-nationale.html [consulté le 26 juin 2017]

9. V. notamment TA Montreuil, 22 nov. 2011, n 1012015, établissant la légalité d'une telle prescription de neutralité religieuse du règlement intérieur d'une école.

10. France. Conseil d'État, Étude demandée par le Défenseur des droits le 20 septembre 2013, adoptée par l'Assemblée générale du Conseil d'État le 19 décembre 2013.

11. Circulaire $\mathrm{n}^{\circ}$ 2012-056 du 27 mars 2012, Orientations et instructions pour la préparation de la rentrée 2012, NOR : MENE1209011C.

12. «Il est recommandé de rappeler dans le règlement intérieur que les principes de laïcité de l'enseignement et de neutralité du service public sont pleinement applicables au sein des établissements scolaires publics. Ces principes permettent notamment d'empêcher que les parents d'élèves ou tout autre intervenant manifestent, par leur tenue ou leurs propos, 
Pour ce qui concerne les stagiaires GRETA, les derniers développements relatifs au statut juridique des interdictions de pénétrer au sein des lycées où se déroulent leur formation professionnelle opposées à des jeunes femmes voilées (i.e. sont-elles légales ?) illustrent que non seulement c'est une interprétation spatiale (l'école comme lieu) et non statutaire (l'élève comme usager du service public de l'éducation) qui prévaut, mais encore qu'elle l'érige en lieu unifié, homogène dans sa pureté aseptique par rapport au religieux. En mars 2009, une note de la direction des affaires juridiques du ministère de l'Éducation nationale allait dans le sens des établissements fermant leurs portes aux stagiaires GRETA voilées, au motif que la coexistence, au sein d'un même lycée, de deux publics distincts au regard de la loi de 2004 (un public de lycéens soumis à l'obligation de neutralité et un public de stagiaires non soumis) serait constitutive d'une rupture d'égalité ${ }^{13}$. Ici et là, les juges qui ont été saisis de recours contentieux n'ont pas toujours suivi ce raisonnement - et les décisions de justice sont contrastées ${ }^{14}$. Mais la dernière en date mérite d'être lue de près, qui valide l'exclusion d'une stagiaire GRETA portant le voile au motif que «les stagiaires du GRETA étaient amenés à rencontrer les élèves du lycée » et que la "présence simultanée » au sein d'un même établissement d'élèves soumis à la loi de 2004 et «d'une stagiaire du GRETA portant un signe [interdit] » devait alors être comprise comme de nature à « troubler l'ordre dans cet établissement ${ }^{15}$ ». Ce que l'on voit ici émerger c'est en effet la construction juridique de la coexistence (entre deux publics d'usagers soumis à deux régimes juridiques distincts) comme, alternativement, une rupture d'égalité ou un risque de trouble à l'ordre public. Où l'on voit que la frontière que dessine la laïcité est comprise comme destinée à conserver une asepsie de l'espace scolaire contre toute impureté aussitôt perçue comme facteur de trouble.

\section{AU TRAVAIL}

Au travail aussi, la neutralité religieuse gagne du terrain. Nul besoin de revenir ici sur l'affaire Baby Loup ${ }^{16}$; mais si ses suites strictement judiciaires

leurs convictions religieuses, politiques ou philosophiques lorsqu'ils accompagnent les élèves lors des sorties et voyages scolaires. » : ibid.

13. Note de la directrice des affaires juridiques relative à l'applicabilité des dispositions de l'article L. 141-5-1 aux stagiaires des GRETA, Ministère de l'Éducation nationale, 10 mars 2009.

14. Jugeant illégales les décisions de refus d'accès opposées à des stagiaires GRETA voilées : TA Paris, 5 nov. 2010, nº 0905232 et TA Caen, 5 avr. 2013, n 1200934.

15. CAA Paris, 12 oct. 2015, n 14 PA00582 [nous soulignons].

16. V. in fine, Cass., ass. plén., 25 juin 2014, n 13-28.369. 
ont été relativement cantonnées ${ }^{17}$, il n'en va pas de même de ce qui s'est passé à la faveur de l'affaire, que ce soit au plan jurisprudentiel, législatif ou politique.

Au plan jurisprudentiel, il importe de souligner l'autre arrêt du 19 mars 2013 - l'arrêt CPAM de Saint Denis ${ }^{18}$, qui marque là aussi une conquête majeure de la frontière de la laïcité sur un territoire nouveau : celui des personnels de droit privé travaillant au sein d'organismes privés chargés d'une mission de service public. Jusque-là, nulle obligation de neutralité religieuse pour eux car seuls les fonctionnaires et agents publics étaient assujettis à la règle précisée dans l'avis Mlle Marteaux rendu par le Conseil d'État en $2000^{19}$. La question était statutaire : les personnels relevant d'un statut de droit public, parce qu'ils incarnent la puissance publique, étaient seuls soumis à la neutralité religieuse. Avec l'arrêt rendu par la Cour de cassation en mars 2013, la question est transformée : la neutralité n'est plus exigée des personnels en fonction de leur statut (sont-ils fonctionnaires ou agents publics?), mais en fonction du point de savoir si leur employeur est, de près ou loin, associé à une mission de service public. Si c'est le cas, une règle de neutralité religieuse dans l'entreprise est possible ${ }^{20}$. Et les suites de l'arrêt ne se sont pas fait attendre, qui ont vu nombre de personnes morales de droit privé chargées de mission de service public (qui existent en nombre très important) inclure,

17. L'arrêt final rendu par l'assemblée plénière de la Cour de cassation est en effet fortement circonstanciel et ne saurait que malaisément être lu comme un arrêt de principe : la solution retenue (i.e. la légalité du licenciement de Mme Afif) repose en effet sur la conjonction de facteurs très spécifiques, à savoir la petite taille de la crèche et le fait que chacun-e des 18 employé-es « étaient ou pouvaient être en relation directe avec les enfants et leurs parents".

18. Cass. soc., 19 mars 2013, $\mathrm{n}^{\circ} 12-11.690$.

19. CE, avis, 3 mai 2000, n 217017 : Mlle Marteaux, « Si les agents du service de l'enseignement public bénéficient comme tous les autres agents publics de la liberté de conscience qui interdit toute discrimination dans l'accès aux fonctions comme dans le déroulement de la carrière qui serait fondée sur leur religion, le principe de laïcité fait obstacle à ce qu'ils disposent, dans le cadre du service public, du droit de manifester leurs croyances religieuses; Il n'y a pas lieu d'établir une distinction entre les agents de ce service public selon qu'ils sont ou non chargés de fonctions d'enseignement; Il résulte de ce qui a été dit ci-dessus que le fait pour un agent du service de l'enseignement public de manifester dans l'exercice de ses fonctions ses croyances religieuses, notamment en portant un signe destiné à marquer son appartenance à une religion, constitue un manquement à ses obligations. »

20. V. l'arrêt : « [...] les principes de neutralité et de laïcité du service public sont applicables à l'ensemble des services publics, y compris lorsque ceux-ci sont assurés par des organismes de droit privé et que, si les dispositions du code du travail ont vocation à s'appliquer aux agents des caisses primaires d'assurance maladie, ces derniers sont toutefois soumis à des contraintes spécifiques résultant du fait qu'ils participent à une mission de service public, lesquelles leur interdisent notamment de manifester leurs croyances religieuses par des signes extérieurs, en particulier vestimentaires. »[nous soulignons]. 
dans leurs règlements intérieurs, des règles générales de neutralité des personnels. Frontière conquérante de la laïcité qui englobe désormais un bien plus grand nombre de travailleurs que sous l'empire de la règle ancienne.

On le sait, l'affaire Baby Loup, qui aura largement contribué à la saillance de la question du fait religieux dans l'entreprise, a également connu des suites législatives. Le temps de l'«affaire » elle-même, de nombreuses propositions de loi visant à permettre aux entreprises de prescrire la neutralité religieuse de leurs personnels avaient été déposées et parfois débattues ${ }^{21}$. La « loi Travail » du 8 août 2016 est l'aboutissement de ces initiatives, qui consacre un nouvel article L. 1321-2-1 du Code du travail aux termes duquel : " Le règlement intérieur peut contenir des dispositions inscrivant le principe de neutralité et restreignant la manifestation des convictions des salariés si ces restrictions sont justifiées par l'exercice d'autres libertés et droits fondamentaux ou par les nécessités du bon fonctionnement de l'entreprise et si elles sont proportionnées au but recherché. » Si l'on ne dispose pas encore du recul suffisant pour prendre la mesure des changements que cette disposition entraînera dans les faits, il faut souligner leur importance de principe. Dans le régime antérieurement applicable, le Code du travail faisait prohibition de principe au règlement intérieur de prescrire la neutralité religieuse des personnels, réputés conserver la jouissance de leurs droits fondamentaux (et notamment, de la liberté religieuse) dans l'emploi ; simplement, des restrictions aux libertés restaient possibles si elles étaient justifiées par les spécificités d'un poste de travail donné et demeuraient proportionnées. La nouvelle formulation est bien éloignée de ce régime, qui permet au contraire à l'entreprise, par principe, de requérir la neutralité religieuse des personnels. Il s'agit donc ici encore d'une évolution juridique qui tend à attraire l'entreprise, de manière quasi généralisée, dans le champ des espaces pouvant valablement être gouvernés par une règle de neutralité religieuse.

Il faut dire que, au plan politique et social, il existe depuis plusieurs années désormais de nombreux signes de la conquête de l'espace «entreprise » par la frontière « laïcité ». Nul ne peut ignorer aujourd'hui la saillance politique et médiatique de la question du fait religieux en entreprise ${ }^{22}$. C'est que la

21. Lemaire E., «La laïcité "répressive" : l'exemple du traitement de l'affaire Baby Loup au Parlement », in Giraudeau G., Bargues C., Haupais N. (dir.), Le fait religieux dans la construction de l'État, Paris, Pedone, 2016, p. 131.

22. V. les études de l'Observatoire du fait religieux en entreprise : http://grouperandstad.fr/ wp-content/uploads/2016/09/cp-exxtude-2016-fait-religieux-en-entreprise-1.pdf ; http:// wellcom.fr/presse/randstad/2015/04/etude-fait-religieux-entreprise/ [consultés le 26 juin 2017]. 
question est devenue si sensible qu'elle reçoit une attention sociale, médiatique et politique sans précédent - et probablement, aussi, qu'elle encourage les pratiques-tests. On songe par exemple à la politique de laïcité, largement publicisée en dépit de son illégalité probable ${ }^{23}$, mise en œuvre au sein de l'entreprise Paprec ${ }^{24}$ depuis $2014^{25}$. On songe encore à l'argumentation mise en avant par l'entreprise Micropole pour justifier le licenciement d'une salariée voilée : les préférences de la clientèle. Sans préjuger de la résolution juridictionnelle de cette affaire, dans le cadre de laquelle la Cour de cassation a posé une question préjudicielle à la Cour de justice de l'Union européenne ${ }^{26}$, il importe de souligner le spectaculaire bond en arrière que fait subir au projet même qui fonde l'existence d'un droit de la non-discrimination l'invocation, par une entreprise, de l'argument des préférences de la clientèle pour justifier une pratique discriminatoire (ici, un licenciement fondé sur la religion de la salariée) ${ }^{27}$. On ne saurait oublier en effet que les "préférences de la clientèle » comptaient parmi les arguments fréquemment mis en avant dans l'Amérique ségrégationniste par les diners et autres commerces refusant les Noirs ou leur réservant des espaces spécifiques ; et c'est précisément l'illégitimité de l'argument que veulent pointer les sit-in organisés par des activistes noirs américains selon le modèle initié par les Greensboro Four qui, à partir des années 1950, compteront parmi les actions pacifistes de revendication d'un Civil Rights Act qui verra le jour en $1964^{28}$.

23. Jusqu'à ce qu'interviennent, coup sur coup, la loi Travail du 8 août 2016 (V. supra) et les arrêts de la CJUE du 14 mars 2017 (Achbita, C-157/15 et Bougnaoui, C-188/15) qui, tous deux, rendent juridiquement possible le choix pour une entreprise de prescrire une stricte politique de neutralité à ses personnels.

24. Charte de la laïcité et de la diversité de Paprec Group : https://www.paprec.com/fr/groupe/ ressources-humaines/charte-laicite-diversite [consulté le 26 juin 2017], et notamment l'article 5 de la charte : « La laïcité en entreprise implique que les collaborateurs ont un devoir de neutralité : ils ne doivent pas manifester leurs convictions politiques ou religieuses dans l'exercice de leur travail. »

25. Gallet L., «Paprec : une charte de la laïcité abusive ? », L'Express, 10 févr. 2014 : http:// lentreprise.lexpress.fr/rh-management/droit-travail/paprec-une-charte-de-la-laicite-abusive_1512086.html [consulté le 26 juin 2017].

26. Cass. soc., 9 avril 2015, n 13-19.855. V. CJUE, Aff. C-188/15, Asma Bougnaoui et ADDH c/ Micropole SA (V. également une affaire belge soulevant des questions proches: CJUE, Aff. C-157/15, Samira Achbita).

27. Wolmark C., «L'entreprise n'est pas un établissement scolaire », RDT 2009, p. 488.

28. Une seconde affaire, belge, portée devant la CJUE soulève aussi des questions de première importance et notamment celle de la légitimité du choix patronal de la neutralité religieuse comme "image de marque », image commerciale de l'entreprise. Pour une critique des conclusions présentées par J. Kokott dans cette affaire, on se permet de renvoyer à Hennette Vauchez S., Wolmark C., " Plus vous discriminez, moins vous discriminez », SSL, $\mathrm{n}^{\circ} 1728,2016$, p. 5-8. 


\section{DANS LA RUE}

Mais, au-delà de l'école et du travail, c'est encore la rue - ou, plus exactement, l'espace public - qui constitue désormais une des frontières spatiales de la laicité. Certes, la loi du 11 octobre 2010 relative à l'interdiction de la dissimulation du visage dans l'espace public est désormais présentée, y compris par le gouvernement français lorsqu'il est amené à la défendre (par exemple en 2014 devant la Cour européenne des droits de l'homme), comme un texte justifié par des impératifs de sécurité publique. Il ne fait pourtant pas de doute que la motivation principale derrière le texte était le souhait, exprimé tant par la mission d'information parlementaire qui a précédé le vote de la loi $^{29}$ que par l'adoption de la résolution «sur l'attachement au respect des valeurs républicaines face au développement de pratiques radicales qui y portent atteinte ${ }^{30} »$ ou encore les intentions du gouvernement, de proscrire, sur le territoire de la République, le port du voile intégral, réputé porter atteinte au «vivre ensemble ${ }^{31} »$. D'ailleurs, quand bien même le titre comme le contenu de la loi sont de formulation neutre, en ce sens qu'ils s'appliquent à tous et visent la dissimulation du visage sans qu'aucune référence soit faite à la dissimulation du visage par un vêtement religieux, la décision du Conseil constitutionnel en date du 7 octobre 2010 révèle la réelle portée du texte, de par la formulation de la réserve de constitutionnalité tenant à l'observation que son application ne saurait porter atteinte à la liberté religieuse ${ }^{32}$. L'espace public, qui reçoit pour l'occasion une définition juridique ${ }^{33}$, se voit donc transformé en nouvelle frontière de la laicité, qui interdit ici la couverture du visage.

29. Rapport d’information sur la pratique du port du voile intégral sur le territoire national, Paris, Assemblée nationale, 26 janv. 2010.

30. Adoptée le 11 mai 2010 par l'Assemblée nationale, sur proposition de M. JeanFrançois Copé.

31. V. le courrier adressé par le Premier ministre M. François Fillon au Conseil d'État au moment de lui demander un avis sur les fondements juridiques possibles d'une interdiction que le gouvernement souhaite "aussi large que possible». Le courrier figure dans l'étude remise par le Conseil d'État en mars 2010 : Étude relative aux possibilités juridiques d'interdiction du port du voile intégral, 30 mars 2010 : http://www.conseil-etat.fr/content/ download/1731/5221/version/1/file/etude_vi_30032010.pdf [consulté le 26 juin 2017].

32. Cons. const., déc. 7 oct. 2010, n 2010-613 DC : « que, toutefois, l'interdiction de dissimuler son visage dans l'espace public ne saurait, sans porter une atteinte excessive à l'article 10 de la Déclaration de 1789, restreindre l'exercice de la liberté religieuse dans les lieux de culte ouverts au public.»

33. Bui-Xuan O. (dir.), Droit et espace(s) public(s), Paris, LGDJ, 2012. 


\section{LA LAÏCITÉ COMME NEW FRONTIER : CONQUÊTE DE L'OUEST ?}

Mais par-delà leurs dimensions spatiales, ces nouvelles frontières de la laïcité sont porteuses de sens et ont un contenu matériel. À mesure que le principe juridique de laïcité se substantialise, ces frontières disent quelque chose de l'identité des personnes et groupes de personnes qui sont placées de part et d'autre de leurs contours. En effet, la prohibition de manifester ses convictions religieuses - à l'école et autour de l'école, au travail, dans la rue... - peut aussi être lue comme une injonction à apparaître sous un certain jour; et c'est la norme qui est exprimée en creux vers laquelle on souhaite ici porter le regard. En ce sens, la laïcité ne se borne pas à soumettre des personnes à des obligations de neutralité religieuse; plus encore, elle véhicule un discours substantiel. Ce discours substantiel est riche et on peut l'appréhender de différentes manières ; on fait le choix ici d'insister sur ce qu'il dit sur le séculier et le religieux.

Rien mieux que la question de l'interprétation, puis de la qualification, des signes d'appartenance à la religion ne permet de saisir ce qui se joue dans les obligations de neutralité religieuse qui sont aujourd'hui déduites du principe de laïcité. C'était en effet une difficulté prévisible de la loi du 15 mars 2004 sur les signes religieux à l'école : qu'est-ce qu'un signe religieux? On le sait, aujourd'hui, les signes qui précisément ont pu être choisis pour leur vocation naturelle à ne pas s'attirer les foudres de la loi sont aujourd'hui régulièrement attraits dans son champ d'application : bandanas ${ }^{34}$, jupes longues ${ }^{35}$, bonnets de laine ${ }^{36} \ldots$ fondent valablement l'exclusion d'élèves qui ne revêtent pas le voile mais accompagnent le port de ces autres signes d'un comportement qui permet aux autorités scolaires, avec l'approbation du juge, de les considérer comme ne respectant pas la loi. Bien au-delà du signe religieux lui-même, c'est aujourd'hui l'intransigeance des élèves dans le refus de retirer un signe, voire une certaine manière de se conformer à la loi (cf. les jeunes filles qui retirent leur voile au dernier moment et le revêtent aussitôt quitté le périmètre scolaire) qui sont réputées constituer, typiquement, des comportements appelant l'application de la loi de 2004. Ce glissement de l'évaluation du signe à celle du comportement de l'élève est capital, qui atteste le fait qu'on ne cherche plus ici tant un espace scolaire expurgé de signes religieux que,

34. CE, 5 déc. 2007, n 295671, M. et Mme A.

35. CE, ord. réf., 19 mars 2013, n 366749. - V. aussi, en 2015, une décision de renvoi fondée sur le port d'une jupe trop longue : SAUvagEt B., "Une jupe pas très "laïque" provoque l'exclusion d'une collégienne », Libération, 28 avril 2015.

36. CE, 10 juin 2009, $\mathrm{n}^{\circ} 306833$. 
dans les termes mêmes de la directrice des affaires juridiques du ministère de l'Éducation nationale, une "adhésion aux valeurs de la République ${ }^{37}$ ». Les élèves peuvent bien dès lors respecter les termes de la loi, c'est insuffisant : on attend d'elles qu'elles adhèrent au projet qui la fonde. Mais on connaît la fragilité de ces mesures et des raisonnements qui les sous-tendent ; elle est au moins double: technique et théorique.

Techniquement, la qualification du signe fait encourir à tout moment le risque de l'arbitraire : à partir de quand une jupe est-elle trop longue ? Qu'est-ce qui permet de «lire » un bandana comme le substitut d'un voile? Difficile de ne pas évoquer ici la spectaculaire affaire estivale du burkini, qui a poussé l'interrogation dans ses retranchements quasi comiques : comment différencier le burkini de la combinaison de plongée, ou encore de n'importe quelle tenue arborée par celle qui aura oublié son maillot de bain au vestiaire ? Faut-il entreprendre de mesurer le pourcentage de peau dénudée sur la plage et revenir, à l'envers, au temps des gendarmes de Saint-Tropez entendant dévider leurs carnets de contraventions à souche en pourchassant les nudistes?

Mais si ces difficultés sont réelles et non seulement risibles, c'est parce qu'elles reposent, au fond, sur une question théorique majeure qu'est celle de l'interprétation d'un signe. Or l'opération d'interprétation elle-même ne peut pas ne pas procéder, au moins pour partie, de l'interprète. En ce sens, il n'y a pas de vérité objective du signe et la signification qui lui est prêtée est nécessairement partiellement tributaire du regard qui le décrypte. D’ailleurs, dans les discours visant l'interdiction du voile, on constate qu'il est tantôt interprété - et condamné - comme un signe religieux (pour exclure des jeunes femmes de l'école en application de la loi de 2004), tantôt au contraire comme un signe politique. Ainsi, nombre d'acteurs politiques et juridiques ont considéré, au cours des dernières années, que « le port du voile n'est pas un signe de piété ou de modestie ; c'est un acte militant motivé par une authentique propagande fondamentaliste ${ }^{38}$ " ou que «porter le voile islamique n'est pas un geste religieux mais politique ${ }^{39} »$.

C'est d'ailleurs la raison pour laquelle l'application des règles relatives à la laiicité, bien plus qu'elle ne procède d'une distinction entre ce qui est religieux et ce qui ne l'est pas, entretient, produit et reproduit sans cesse cette

37. Moreau C. (DGESCO), «Bilan de l'application de la loi du 15 mars 2004 », in France. ObSeRVATOIRE DE LA LaḯcitÉ, Rapport annuel 2013-2014, p. 79.

38. Alain Juppé, cité in Fernando M., The Republic Unsettled. Muslim French and the Contradictions of Secularism, London, Duke University Press, 2014, p. 142.

39. Françoise Hostalier (députée UMP, inspectrice de l'Éducation nationale), citée in FERNANDO M., op. cit. 
distinction. La récente intervention du Conseil d'État sur les crèches de la Nativité en fournit un exemple emblématique ${ }^{40}$. Le Conseil d'État était saisi de recours contre des décisions prises par des collectivités locales d'installer, dans des bâtiments publics, des crèches de la Nativité - et la question de leur compatibilité avec l'article 28 de la loi du 9 décembre 1905 était notamment posée $^{41}$. Suivant - au moins pour partie - les conclusions présentées devant lui par la rapporteure publique Aurélie Bretonneau, il fit le choix d'une lecture très culturaliste de la pratique qui était en cause ${ }^{42}$. Il s'appuie dans un premier temps sur le fait que la loi de 1905 n'interdit l'apposition d'emblèmes que pour autant qu'ils ont une vocation permanente - mais non nécessairement ceux qui seraient temporaires. Il considère ensuite, et en outre, que : « une crèche de Noël est une représentation susceptible de revêtir une pluralité de significations. Il s'agit en effet d'une scène qui fait partie de l'iconographie chrétienne et qui, par là, présente un caractère religieux. Mais il s'agit aussi d'un élément faisant partie des décorations et illustrations qui accompagnent traditionnellement, sans signification religieuse particulière, les fêtes de fin d'année. " Dès lors, c'est à une véritable casuistique juridique que la Haute juridiction invite les autorités administratives et les juges du fond, en posant la règle que « eu égard à cette pluralité de significations, l'installation d'une crèche de Noël, à titre temporaire, à l'initiative d'une personne publique, dans un emplacement public, n'est légalement possible que lorsqu'elle présente un caractère culturel, artistique ou festif, sans exprimer la reconnaissance d'un culte ou marquer une préférence religieuse ». Elle précise en outre que «pour porter cette dernière appréciation, il y a lieu de tenir compte non seulement du contexte, qui doit être dépourvu de tout élément de prosélytisme, des conditions particulières de cette installation, de l'existence ou de l'absence d'usages locaux, mais aussi du lieu de cette installation». En ce sens, elle établit qu'a priori, l'installation d'une crèche dans l'enceinte de bâtiments publics sièges d'une collectivité publique ou d'un service public est contraire au principe de neutralité des personnes publiques - mais laisse tout de même ouverte la voie de « circonstances particulières permettant de lui reconnaître un caractère culturel, artistique ou festif », tandis que dans les autres emplacements publics, l'installation est

40. V. l'article de Hélène Pauliat dans ce même numéro.

41. Art. 28 de la loi du 9 décembre 1905 : «Il est interdit, à l'avenir, d'élever ou d'apposer aucun signe ou emblème religieux sur les monuments publics ou en quelque emplacement public que ce soit, à l'exception des édifices servant au culte, des terrains de sépulture dans les cimetières, des monuments funéraires, ainsi que des musées ou expositions. »

42. V. les arrêts : CE, ass., 9 nov. 2016, n³95122, Fédération départementale des libres penseurs de Seine-et-Marne et $\mathrm{n}^{\circ}$ 395223, Fédération de la libre pensée de Vendée. V. aussi DutHeILLET de Lamothe V., Odinet G., « La crèche entre dans les tables », AJDA 2016, p. 2375. 
a priori possible car a priori festive, sauf à ce qu'on puisse y déceler un acte de prosélytisme ou de revendication d'une opinion religieuse. En d'autres termes, ce que révèlent ces importants arrêts d'assemblée, c'est à tout le moins que le juge administratif est disposé à prêter une attention fine et à faire reposer la règle d'interdiction sur des distinctions subtiles - distinction entre signification religieuse et signification « culturelle », distinction entre bâtiments publics et emplacements publics -, attitude qui se situe aux antipodes de celle qu'il adopte dans son travail d'interprétation de la loi du 15 mars 2004, fondée sur une lecture rigoureusement mécanique des «signes ou tenues par lesquels les élèves manifestent ostensiblement une appartenance religieuse » qui lui permet d'y englober, pêle-mêle, jupes trop longues et bandeaux de tête trop larges.

Le contraste entre la jurisprudence administrative relative à la loi de 2004 et celle qui vient d'être arrêtée relativement à l'article 28 de la loi du 9 décembre 1905 établit bien que la jurisprudence relative à la laïcité ne découle pas d'une distinction entre le religieux et le séculier mais qu'elle la produit : en définissant toujours le voile (et même au-delà, le bandana, la jupe, etc.) comme religieux, et en exigeant des élèves non seulement le respect mais encore l'adhésion à la règle de neutralité religieuse qui s'impose à eux, la jurisprudence administrative fait émerger une compréhension de l'école laique comme expurgée du voile (et, occasionnellement, du turban sikh); en définissant la crèche de la Nativité comme tantôt religieuse, tantôt culturelle (festive), elle fait émerger une compréhension des bâtiments et emplacements publics, eux aussi laïques, comme non nécessairement aseptisés.

Lire la laïcité comme frontière, non seulement spatiale mais aussi matérielle, permet aussi de voir la multiplicité des terrains sur lesquelles elle fait irruption. On l'a vu, la frontière matérielle de la laïcité peut être lue comme produisant la distinction entre le séculier et le religieux. Mais elle intervient encore sur d'autres terrains, et joue un rôle croissant dans la distinction entre les nationaux et les étrangers, entre la République et ses antonymes...

Le rôle de la laïcité dans la fabrication de l'identité nationale se donne largement à voir aujourd'hui dans le droit de la nationalité ou dans le droit des étrangers. Ainsi, différents travaux ont montré combien, par exemple, la déclaration acquisitive de nationalité était devenue un terrain privilégié de nouveaux usages du principe de laïcité ${ }^{43}$ largement pétris d'injonctions

43. Sur l'arrêt fondateur de 2008, V. Koussens D., "Sous l'affaire de la burqa, quel visage de la laïcité française ? », Sociologie et Sociétés, vol. 41, n² 2, 2009, p. 327-347, spéc. p. 336. 
à des modes de vie et favorables à un discours sur soi, sur le national ${ }^{44}$; d'autres travaux, plus ethnographiques, ont pu établir combien le travail mené dans les bureaux des naturalisations ${ }^{45}$ ou l'organisation des cérémonies de naturalisation ${ }^{46}$, faisaient eux aussi une large part à des références à la laïcité. Plus encore, le droit des étrangers est à l'avenant - alors même que, par hypothèse, toute référence ici à l'assimilation ou l'intégration devrait être moins impérieuse. La consolidation du contrat d'accueil et d'intégration (aujourd'hui contrat d'intégration républicaine) marque une étape importante, dans la mesure où, comme l'établit une thèse récente consacrée à la genèse et la mise en ouvre de ce contrat ${ }^{47}$, sa conclusion s'accompagne de nombreux discours et démarches administratives dans lesquels la référence à la laïcité est centrale... jusqu'à emporter des reformulations de la devise républicaine à laquelle la laïcité est ajoutée comme quatrième pied...

Plus généralement, la laïcité opère bien souvent comme une métonymie de la République. Mais bien sûr, de telles évolutions de la structure du débat public sur la laïcité commandent une extrême vigilance, tant elles reposent sur l'axiologisation forte de notions floues. Comment poser la laïcité comme emblème de la République à l'heure même où les frontières de la première sont plus mouvantes et polémiques qu'elles ne l'avaient été depuis de nombreuses décennies, et que la seconde fait bien souvent figure encore de promesse plus que de réalisation?

Sur l'ensemble de la jurisprudence relative à l'article 21-4 du Code civil, V. HennEtTE VAuCHEZ S., "Genre et religion : le genre de la nouvelle laïcité », in REGINE, HennetTe VAuchez S., Pichard M., Roman D. (dir.), La loi \& le genre. Études critiques de droit français, Paris, Éd. du CNRS, 2014, p. 715-731.

44. Particulièrement emblématiques de ce point de vue sont les conclusions prononcées par les rapporteurs publics dans ces affaires; on renverra plus particulièrement à celles prononcées par Emmanuelle Prada-Bordenave sur CE, 27 juin 2008, n 286798 et Xavier Domino sur CE, 27 nov. 2013, n 365587.

45. Hajjat A., Les frontières de l'identité nationale, Paris, La Découverte, 2012.

46. Mazouz S., Fassin D., «Qu'est-ce que devenir français?», Revue française de sociologie, $\mathrm{n}^{\circ}$ 4, vol. 48, 2007, p. 723-750.

47. Gourdeau C., L'intégration des étrangers sous injonction. Genèse et mise en ouvre du CAI, Thèse socio-ethnologie, Paris 8, 2015. 